\title{
Association of brachial-ankle pulse wave velocity with atherosclerosis and presence of coronary artery disease in older patients
}

This article was published in the following Dove Press journal:

Clinical Interventions in Aging

20 August 2015

Number of times this article has been viewed

\author{
Chang-Min Chung ${ }^{1,2}$ \\ Yu-Hsiang Tseng ${ }^{3}$ \\ Yu-Sheng Lin ${ }^{3}$ \\ Jen-Te $\mathrm{Hsu}^{4}$ \\ Po-Chang Wang ${ }^{3}$
}

'School of Traditional Chinese Medicine, College of Medicine, Chang Gung University, Taoyuan County, ${ }^{2}$ Chang Gung University of Science and Technology, Chiayi County,

${ }^{3}$ Division of Cardiology, Chang Gung Memorial Hospital, Chiayi, ${ }^{4}$ Department of Medicine, College of Medicine, Chang Gung University,

Taoyuan County, Taiwan
Correspondence: Chang-Min Chung Division of Cardiology, Chiayi Chang Gung Memorial Hospital, 6, Sector West Chai-Pu Road, Pu-Tz City, Chai Yi Hsien, Taiwan

Tel +88653621000 ext 2854

Fax +886 53623005

Email cmchung02@hotmail.com
Objective: Brachial-ankle pulse wave velocity (baPWV) is a simple and reproducible measure of arterial stiffness and is extensively used to assess risk of cardiovascular disease in Asia. We examined whether baPWV was associated with coronary atherosclerosis and presence and extent of coronary artery disease (CAD) in older patients with chest pain.

Methods: This cross-sectional study enrolled 370 consecutive patients $>65$ years old who underwent baPWV measurement and elective coronary angiogram for suspected CAD at a single cardiovascular center, between June 2013 and July 2014.

Results: In addition to diabetes mellitus and body mass index, baPWV was one of the statistically meaningful predictors of significant CAD (diameter of stenosis $>50 \%$ ) in a multivariate analysis. When the extent of CAD was classified as nonsignificant or significant CAD (ie, one-, two-, and three-vessel disease), there was a significant difference in baPWV between the significant and nonsignificant CAD groups, but not between the three significant CAD groups. Multivariate linear regression analyses showed that the number of diseased vessels and baPWV were both significantly associated with the SYNTAX (SYNergy between percutaneous coronary intervention with TAXus and cardiac surgery) score. The cutoff value of baPWV at $1,874 \mathrm{~cm} / \mathrm{s}$ had a sensitivity of $60.1 \%$, specificity of $70.8 \%$, and area under receiver operating characteristic curve of 0.639 in predicting CAD.

Conclusion: Arterial stiffness determined by baPWV was associated independently with CAD severity, as assessed by angiography and the SYNTAX score in older patients with chest pain. As a result, increased arterial stiffness assessed by baPWV is associated with the severity and presence of CAD in older patients.

Keywords: brachial-ankle pulse wave velocity, arterial stiffness, coronary artery disease, SYNTAX score

\section{Introduction}

Coronary artery diseases (CAD) are among the leading causes of morbidity and mortality worldwide. One of the current strategies for reducing CAD involves the implementation of prevention strategies among individuals who are at an intermediate risk for $\mathrm{CAD}$ and who would benefit most from CAD prevention therapy. ${ }^{1}$ Arterial stiffness has been reported to be related to atherosclerosis and CAD and has been considered a strong independent predictor of coronary events and cardiovascular mortality among several patient groups. Arterial stiffness can be evaluated by the measurement of brachial-ankle pulse wave velocity (baPWV) along the arterial tree. Unlike carotid-femoral pulse wave velocity (cfPWV), which is a measure of central arterial stiffness, baPWV is a combined measure of central and peripheral arterial stiffness $^{2}$ and is an effective index of arterial stiffness of large arteries. baPWV requires 
the application of cuffs on the four extremities, is convenient to measure in a clinic, requires little technical expertise, and, unlike cfPWV, does not require exposure to the inguinal region. It is therefore widely used for noninvasive assessment of atherosclerosis. ${ }^{3}$

The predictive value of baPWV for cardiovascular events has been reported in high-risk populations, including for patients undergoing chronic hemodialysis ${ }^{4,5}$ and patients with acute coronary syndrome, ${ }^{6}$ diabetes with $\mathrm{CAD},{ }^{7}$ and heart failure. ${ }^{8}$ A meta-analysis of 12 cohort studies confirmed that baPWV was an independent predictor for cardiovascular disease onset and showed that an increase of $1 \mathrm{~m} / \mathrm{s}$ was associated with a $12 \%$ increase in the risk of cardiovascular events. ${ }^{9}$ baPWV is therefore considered reflective of the progression of individual arteriosclerosis and of overall cardiovascular risk.

A number of studies have suggested that there is a relationship between increased PWV and CAD. ${ }^{10-12}$ However, although CAD and arterial stiffness share many risk factors, the correlation between arterial stiffness and the complexity of CAD is not consistent. Arterial stiffness assessed by PWV was shown to be associated with severity of CAD using the SYNTAX (SYNergy between percutaneous coronary intervention with TAXus and cardiac surgery) score only in one study. ${ }^{13}$ The SYNTAX score is an angiographic scoring system developed to aid revascularization decisions and predicts mortality and morbidity in patients irrespective of disease severity. There is a progressive increase in the rigidity of the arterial wall and arterial stiffness as a result of aging. ${ }^{14,15}$ In this study, we retrospectively evaluated the relationship between arterial stiffness assessed by baPWV and the presence and extent of CAD in older patients.

\section{Methods}

\section{Study population}

This study retrospectively enrolled 370 subjects $\geq 65$ years old who were evaluated for ischemic heart disease at the Chiayi Chang Gung Memorial Hospital. Inclusion criteria were presence of ischemic heart disease-like symptoms associated with an abnormal stress test. Exclusion criteria were 1) history of percutaneous coronary intervention, 2) history of myocardial infarction, or 3 ) prior coronary arterial bypass surgery. Patients with a history of severe valvular disease, congenital heart disease, cardiomyopathy, atrial fibrillation, and abnormal conduction disturbances were also excluded. Patients with a history of peripheral artery disease with or without intermittent claudication or ankle brachial index $<0.9$ were excluded from this study. All patients underwent angiograms and baPWV measurements were made from June 2013 to July 2014. The measurement of baPWV and cardiac echography was performed within 1 week prior to angiography. Routine biochemical analysis of the blood and urine were performed. Study patients were evaluated for total cholesterol, high-density lipoprotein cholesterol, low-density lipoprotein cholesterol, plasma triglycerides, fasting blood glucose, uric acid, and serum creatinine after fasting for $\sim 12$ hours. All study protocols were approved by the Chang Gung Medical Foundation Institutional Review Board (IRB102-2739B), and signed informed consent was obtained from all patients.

\section{Definitions}

Diabetes mellitus was defined as a previously diagnosed condition, adherence to a prescribed diet or use of antidiabetic medicine, or a fasting venous blood glucose level of $\geq 126 \mathrm{mg} / \mathrm{dL}$ on two separate occasions. Dyslipidemia was defined as a previously diagnosed condition, use of lipid-lowering agents, elevated plasma total cholesterol $(\geq 200 \mathrm{mg} / \mathrm{dL})$ and/or high triglycerides $(\geq 150 \mathrm{mg} / \mathrm{dL})$, or a low high-density lipoprotein level $(<40 \mathrm{mg} / \mathrm{dL})$.

\section{Assessment of aortic stiffness}

The baPWV measurement protocol has been previously described. ${ }^{2}$ Medications in current use were allowed on the day of examination. Subjects were examined in the supine position at room temperature after 5 minutes in a sedentary position. baPWV was measured using a volume-plethysmographic apparatus (VP-1000; Colin Co. Ltd, Komaki, Japan). Four oscillometric cuffs were wrapped on both the brachia and ankle. They were simultaneously pressurized to the approximate value of the patient's diastolic pressure so that the pulse volume waveforms could be recorded using semiconductor pressure sensors. Two electrocardiogram electrodes were placed on each wrist. These were simultaneously pressurized to the approximate value of the patient's diastolic pressure so that the pulse volume waveforms could be recorded using semiconductor pressure sensors. The time interval between the wave front of the brachial waveform and that of the ankle waveform was defined as the time interval between the brachium and ankle (DTba). The distance between sampling points of the baPWV was calculated automatically according to the height of the patient. The path length from the suprasternal notch to the brachium ( $\mathrm{Lb}$ ) was obtained from superficial measurements and was expressed using the following equation:

$$
\mathrm{Lb}=(0.2195 \times \text { height of the subject }[\mathrm{in} \mathrm{cm}]-2.0734) .
$$


The path length from the suprasternal notch to the ankle (La) was obtained from superficial measurements and was expressed using the following equation:

$\mathrm{La}=(0.8129 \times$ height of the subject $[$ in $\mathrm{cm}]+12.328)$.

baPWV was calculated using the formula:

$$
\text { baPWV }=\frac{\mathrm{La}-\mathrm{Lb}}{\mathrm{DTba}} .
$$

In this study, the left-side baPWV was used for the analyses.

\section{Echocardiographic studies}

Echocardiography was performed by a single sonographer who was blinded to the patient's information. The twodimensional and M-mode images were recorded using an echocardiography machine (PHILIPS IE33) according to the guidelines of the American Society of Echocardiology. ${ }^{16}$ Measurements were made online and records were identified using the participants' initials and the study number. Left ventricular mass (LVM) was quantified using the Devereux formula, ${ }^{17}$ and the LVM index was calculated as the LVM divided by the body surface area.

\section{SYNTAX score and angiographic analysis}

All study patients underwent coronary angiograms via the left radial or brachial artery at the physician's discretion. Coronary angiograms were assessed independently by two physicians who were blinded to the information about the baPWV of individual patients. Significant CAD was defined as $>50 \%$ narrowing of the lumen by visual estimation. Subjects were assigned to the nonsignificant, one-, two-, or three-vessel disease groups based on the presence of a $50 \%$ or greater diameter narrowing for each of the three main coronary arteries (the patients with left main disease were included in the two-vessel disease group). The SYNTAX score for each patient was calculated by a team of two interventional cardiologists. Quantitative coronary angiography was performed in cases where there was disagreement regarding the significance of the lesion, and the lesion was included if there was $\geq 50 \%$ stenosis. On agreement between the two cardiologists, the data were entered into a dedicated software program.

\section{Statistical analysis}

Qualitative variables were expressed as percentages (\%), and quantitative variables were expressed as the mean value \pm standard deviation. Comparison of parametric values between the two groups was carried out by means of a two-tailed Student's $t$-test. Categorical variables were compared by the likelihood-ratio test $\left(w^{2}\right)$ or Fisher's exact test. A multivariate logistic regression analysis was carried out to assess the odds ratios of factors related to significant CAD. Multivariable linear regression analysis was performed for studying the independent correlates of SYNTAX score. A receiver operating characteristic (ROC) curve was used to show a positive correlation between the baPWV in patients with angina. The sum of sensitivity and specificity determined the cutoff values. All statistical analyses were performed using the Statistical Package for the Social Sciences 20.0 statistical package (IBM Corporation, Armonk, NY), and a $P$-value $<0.05$ was considered statistically significant.

\section{Results}

Study patients were classified into the no-CAD or CAD groups. Patient demographics and baseline clinical characteristics are summarized in Table 1. The study population comprised 238 males and 132 females. Patients in the no-CAD group

Table I Demographics and baseline patient characteristics

\begin{tabular}{llll}
\hline & No CAD (n=72) & CAD (n=298) & P-value \\
\hline Male (\%) & 56.9 & 66.4 & 0.085 \\
Age (years) & $71.7 \pm 4.4$ & $72.6 \pm 5.3$ & $0.047^{*}$ \\
BMI (kg/m²) & $26.6 \pm 3.8$ & $25.3 \pm 3.7$ & $0.008^{*}$ \\
BSA (m²) & $1.70 \pm 0.21$ & $1.69 \pm 0.17$ & 0.826 \\
Hypertension (\%) & 71.8 & 76.8 & 0.231 \\
Diabetes (\%) & 23.2 & 44.1 & $0.00 I^{*}$ \\
Smoking (\%) & 10.0 & 19.7 & $0.036^{*}$ \\
Dyslipidemia (\%) & 53.5 & 68.3 & $0.015^{*}$ \\
CVA (\%) & 10.1 & 5.5 & 0.128 \\
TC (mg/dL) & $173.4 \pm 34.1$ & $179.3 \pm 38.2$ & 0.242 \\
HDL-C (mg/dL) & $43.7 \pm 12.5$ & $41.4 \pm 17.1$ & 0.305 \\
LDL-C (mg/dL) & $104.8 \pm 32.8$ & $107.4 \pm 35.2$ & 0.588 \\
Triglyceride (mg/dL) & $129.9 \pm 66.3$ & $156.5 \pm 109.4$ & $0.01 I^{*}$ \\
Creatinine (mg/dL) & $1.3 \pm 1.0$ & $1.7 \pm 1.0$ & 0.473 \\
Uric acid (mg/dL) & $7.1 \pm 1.6$ & $6.6 \pm 1.9$ & 0.065 \\
SBP (mmHg) & $138.3 \pm 20.8$ & $139.6 \pm 21.9$ & 0.635 \\
DBP (mmHg) & $75.1 \pm 12.8$ & $76.3 \pm 12.3$ & 0.488 \\
Hemoglobin (g/dL) & $13.0 \pm 1.9$ & $13.0 \pm 2.1$ & 0.977 \\
EF $(\%)$ & $67.1 \pm 12.6$ & $64.6 \pm 13.0$ & 0.156 \\
LVMI (g/m²) & $119.4 \pm 52.7$ & $114.1 \pm 52.8$ & 0.449 \\
LVH (\%) & 14.3 & 14.2 & 0.556 \\
baPWV (m/s) & $18.33 \pm 3.52$ & $20.22 \pm 4.78$ & $0.002^{*}$ \\
\hline Note $\%$ Denos & &
\end{tabular}

Note: *Denotes significant difference.

Abbreviations: baPWV, brachial-ankle pulse wave velocity; BMI, body mass index; BSA, body surface area; CAD, coronary artery disease; CVA, cerebral vascular accident; DBP, diastolic blood pressure; EF, ejection fraction; LVMI, left ventricular mass index; LVH, left ventricular hypertrophy; HDL-C, high-density lipoprotein cholesterol; LDL-C, low density lipoprotein cholesterol; SBP, systolic blood pressure; TC, total cholesterol. 
had a lower mean age, a lower proportion of diabetes mellitus, smoking, dyslipidemia, and lower triglyceride levels compared to patients in the CAD group. There were no significant differences in body surface area, hypertension, stroke, total cholesterol, low-density lipoprotein cholesterol, high-density lipoprotein cholesterol, blood pressure, serum creatinine, uric acid, hemoglobin, left ventricular ejection fraction or LVM index between the two groups; however, body mass index was significantly lower in the CAD group compared to the no-CAD group. In addition, mean (standard deviation) baPWV was significantly higher in the CAD group compared to the no-CAD group (20.22 [4.78] m/s vs 18.33 [3.52] m/s, $P=0.002)$.

All the factors of significant difference in the univariate analysis and the traditional risk factors of CAD were entered in multivariate logistic regression analysis. Multivariate logistic regression analysis to detect factors significantly associated with CAD showed a significant correlation between diabetes mellitus, body mass index, and baPWV and occurrence of CAD (Table 2). Univariate analysis showed that the SYNTAX score was significantly correlated with age $(\beta=0.161$, $P=0.009), \operatorname{SBP}(\beta=0.238, P<0.001), \mathrm{DBP}(\beta=0.150, P=0.014)$, hemoglobin $(\beta=-0.127, P=0.04$. $)$, number of involved vessels ( $\beta=0.556, P<0.001)$, and baPWV $(\beta=0.637, P<0.001)$ in all the patients (Table 3 ). The association between SYNTAX score and number of involved vessels $(\beta=0.512, P<0.001)$ and baPWV $(\beta=0.597, P<0.001)$ remained significant in multivariate linear regression analyses (Table 3 ).

Figure 1 shows a significant correlation between baPWV and SYNTAX score $\left(R^{2}=0.403, P<0.001\right)$, while Figure 2 shows the association between baPWV and the presence of CAD. Based on the extent of CAD, subjects were classified into one of four groups: nonsignificant stenosis, one-vessel,

Table 2 Multivariate logistic regression analysis of factors associated with coronary artery disease

\begin{tabular}{llll}
\hline & Odds ratio & 95\% confidence interval & P-value \\
\hline Male sex & 1.535 & $0.785-3.005$ & $0.21 \mathrm{I}$ \\
Age & 1.049 & $0.980-1.123$ & 0.167 \\
BMI & 0.919 & $0.852-0.992$ & $0.029 *$ \\
Hypertension & 1.190 & $0.603-2.346$ & 0.616 \\
Diabetes & 2.314 & $1.124-4.765$ & $0.023^{*}$ \\
Smoking & 1.895 & $0.748-4.797$ & 0.177 \\
Dyslipidemia & 1.909 & $0.989-3.687$ & 0.054 \\
Total cholesterol & 1.001 & $0.985-1.017$ & 0.920 \\
HDL & 0.999 & $0.982-1.017$ & 0.929 \\
LDL & 0.999 & $0.984-1.015$ & 0.938 \\
Triglyceride & 1.002 & $0.998-1.007$ & 0.346 \\
baPWV & 1.097 & $1.011-1.191$ & $0.026 *$ \\
\hline Note: & &
\end{tabular}

Note: *Denotes significant difference.

Abbreviations: baPWV, brachial-ankle pulse wave velocity; BMI, body mass index; $\mathrm{Cl}$, confidence interval; HDL, high-density lipoprotein; LDL, low-density lipoprotein. two-vessel, and three-vessel disease. There was a significant difference in baPWV between each of these groups and the no-CAD group. However, there was no significant difference in baPWV between the different CAD groups.

We used ROC curve analysis to evaluate the association between baPWV and CAD and showed a trend toward a positive association between the extent of CAD and baPWV (Figure 3). Using the ROC curve, we determined the optimal cutoff value of baPWV and CAD that could predict the presence of coronary stenosis (Figure 3 ). The cutoff value of baPWV at $1,874 \mathrm{~cm} / \mathrm{s}$ had a sensitivity of $60.1 \%$, specificity of $70.8 \%$, and area under ROC curve of 0.639 in predicting coronary artery stenosis.

\section{Discussion}

This cross-sectional study showed that baPWV was independently and significantly associated with CAD in older patients who had no previous history of ischemic heart disease. Although there was no clinically relevant difference between groups of patients classified according to the severity of CAD, our data showed that baPWV was correlated with a significant increase in the severity and complexity of CAD evaluated by the SYNTAX score, as detected by CAG.

The relevance of central artery stiffness in CAD risk stratification was recently reported. ${ }^{18}$ Although the use of cfPWV, a conventional method for evaluating arterial stiffness, has been established for atherosclerotic diseases, ${ }^{19}$ its use in clinical settings is limited due to technical difficulties. baPWV is a simple alternative and requires short measurement times. In addition, baPWV has been shown to correlate well with cfPWV ${ }^{2}$ and arterial stiffness in an invasive study. ${ }^{20}$ The prognostic value of baPWV has also been well validated in various clinical settings. ${ }^{6,7,9,21}$ baPWV is therefore widely used as a screening tool for the measurement of arterial stiffness in central and peripheral arteries. Because baPWV is a simple index of the severity of arterial stiffness or atherosclerosis, it is appropriate for use in routine examinations as well as for large clinical trials. In addition to the finding that baPWV was closely associated with central arterial stiffness, a positive correlation between baPWV and carotid artery atherosclerosis has also been reported. ${ }^{22}$

$\mathrm{CAG}$ is considered to be the gold standard for diagnosing CAD. The SYNTAX score is an angiographic scoring system that has been shown to be able to aid revascularization decisions. ${ }^{23}$ This system could also predict major adverse cardiac event (MACE) after percutaneous revascularization in patients with multivessel CAD or left main lesion; ${ }^{24}$ however, because $\mathrm{CAG}$ is an invasive examination and a high level of skill is 
Table 3 Univariate and multivariate analysis to evaluate the correlation of SYNTAX score with clinical parameters

\begin{tabular}{|c|c|c|c|c|c|c|}
\hline \multirow[t]{2}{*}{ Parameters } & \multicolumn{3}{|l|}{ Univariate } & \multicolumn{3}{|l|}{ Multivariate } \\
\hline & Coefficients $\beta$ & Tolerance & $P$-value & Coefficients $\beta$ & Tolerance & $P$-value \\
\hline Age & 0.161 & 2.642 & 0.009 & - & - & - \\
\hline BMI & 0.001 & 0.008 & 0.993 & - & - & - \\
\hline BSA & -0.083 & -1.356 & 0.176 & - & - & - \\
\hline TC & 0.067 & 1.064 & 0.288 & - & - & - \\
\hline HDL-C & -0.061 & -0.967 & 0.334 & - & - & - \\
\hline LDL-C & 0.076 & 1.199 & 0.232 & - & - & - \\
\hline Triglyceride & 0.099 & $\mathrm{I} .575$ & 0.116 & - & - & - \\
\hline Creatinine & 0.068 & 1.076 & 0.283 & - & - & - \\
\hline Uric acid & $-0.08 \mathrm{I}$ & -1.184 & 0.238 & - & - & - \\
\hline SBP & 0.238 & 3.981 & $<0.001$ & -0.013 & -0.251 & 0.802 \\
\hline DBP & 0.150 & 2.470 & 0.014 & 0.031 & 0.658 & 0.511 \\
\hline Hemoglobin & -0.127 & -2.029 & 0.043 & 0.008 & 0.198 & 0.843 \\
\hline EF & -0.084 & -1.308 & 0.192 & - & - & - \\
\hline LVMI & 0.021 & 0.349 & 0.728 & - & - & - \\
\hline Vessel no & 0.556 & 10.865 & $<0.001$ & 0.512 & 13.854 & $<0.001$ \\
\hline baPWV & 0.637 & 13.415 & $<0.001$ & 0.597 & 14.832 & $<0.001$ \\
\hline
\end{tabular}

Abbreviations: baPWV, brachial-ankle pulse wave velocity; BMI, body mass index; BSA, body surface area; DBP, diastolic blood pressure; EF, ejection fraction; HDL-C, high-density lipoprotein cholesterol; LDL-C, low density lipoprotein cholesterol; LVMI, left ventricular mass index; SBP, systolic blood pressure; SYNTAX, SYNergy between percutaneous coronary intervention with TAXus and cardiac surgery; TC, total cholesterol; Vessel no, number of involved vessels.

required for accurate measurement, its applicability is limited. Arterial stiffness evaluated by baPWV may reflect vascular health, particularly endothelial dysfunction, ${ }^{25,26}$ which was shown to be an important risk factor for future cardiovascular events and a marker for early atherosclerosis. ${ }^{27}$

baPWV has been suggested to be a marker of cardiovascular risk stratification. ${ }^{21}$ baPWV of $1,635 \mathrm{~cm} / \mathrm{s}$ had a sensitivity of $73 \%$ and specificity of $75 \%$ in detecting complex CAD in

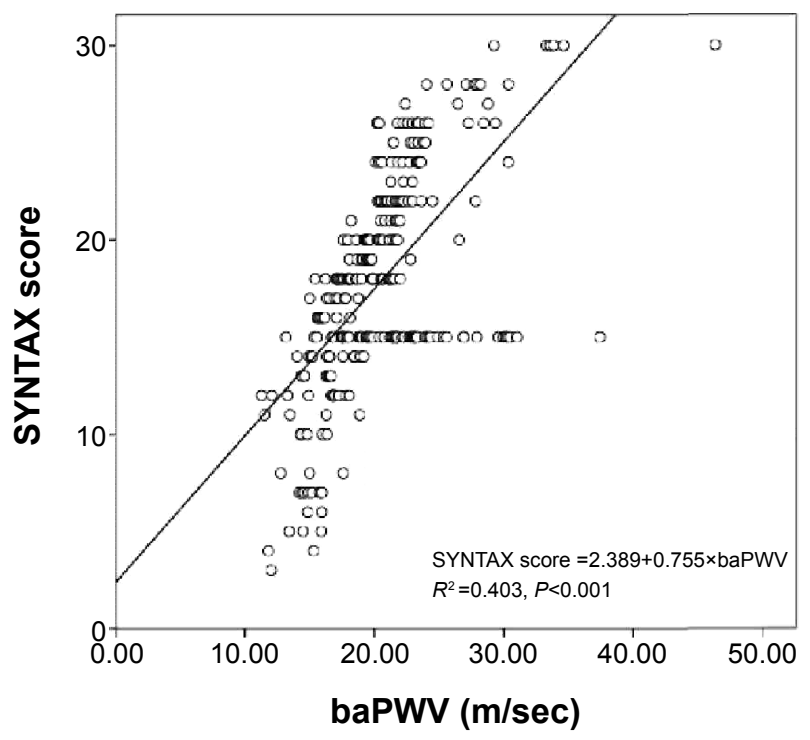

Figure I Linear regression between baPWV and SYNTAX score.

Note: The baPWV was significantly correlated with Syntax score $\left(R^{2}=0.525\right.$, $P<0.001$ ).

Abbreviations: baPWV, brachial-ankle pulse wave velocity; SYNTAX, SYNergy between percutaneous coronary intervention with TAXus and cardiac surgery. diabetic patients. ${ }^{28}$ baPWV $\geq 1,426 \mathrm{~cm} / \mathrm{s}$ was associated with a high stenosis rate in coronary computed tomography angiography in asymptomatic patients, ${ }^{10}$ while baPWV $>1,800 \mathrm{~cm} / \mathrm{s}$ followed a severe coronary event. ${ }^{29}$ Our study revealed that in suspected CAD patients, a baPWV of $1,874 \mathrm{~cm} / \mathrm{s}$ had a sensitivity of $60.1 \%$, specificity of $70.8 \%$, and an AUC of 0.639 in predicting coronary artery stenosis.

The mechanisms underlying the association between baPWV and CAD were investigated. First, elevated PWV

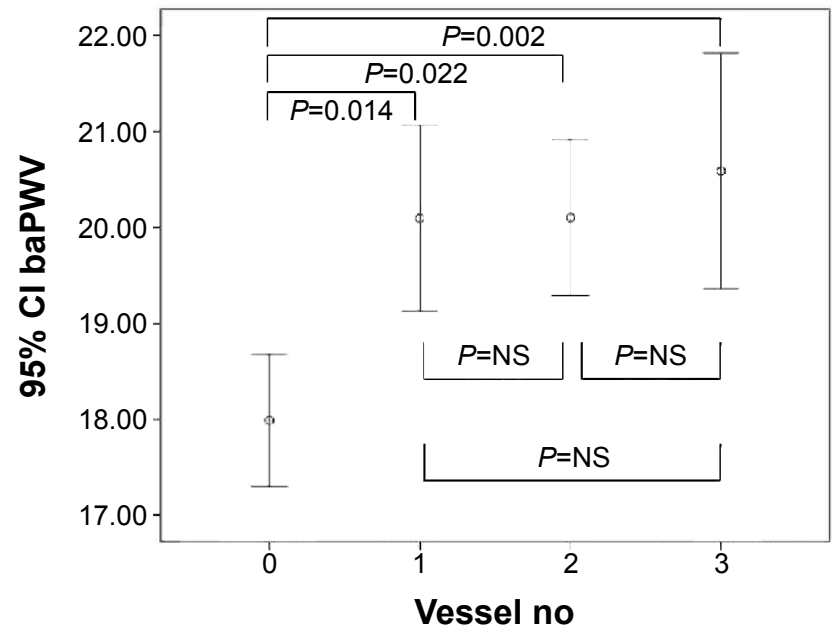

Figure 2 Comparison of baPWV based on the extent of coronary artery disease. Notes: There was no significant difference in baPWV values between the groups except for subjects without significant stenosis. However, there was a linear trend between the number of stenosed vessels and increased baPWV.

Abbreviations: baPWV, brachial-ankle pulse wave velocity; NS, not significant; Vessel no, vessel number; $\mathrm{Cl}$, confidence interval. 


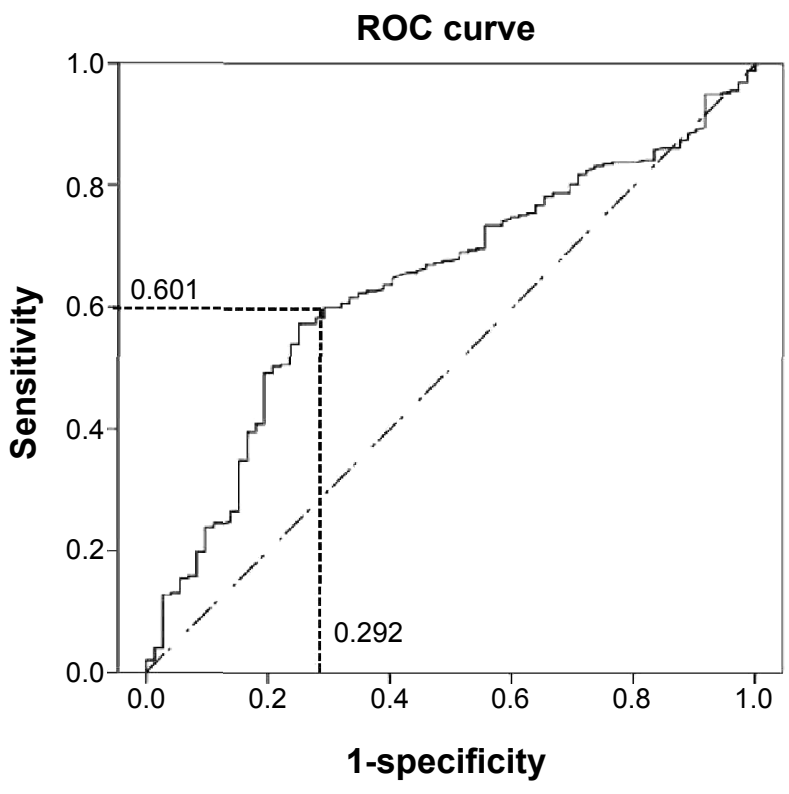

Figure 3 ROC curve between baPWV and the presence/absence of coronary artery disease.

Note: Area under ROC curve of 0.639 predicts coronary artery stenosis Abbreviations: baPWV, brachial-ankle pulse wave velocity; ROC, receiver operating characteristic.

represents increased arterial stiffness. The conventional atherosclerotic risk factors, which are used to determine the Framingham risk score, may contribute synergetically to arterial stiffness and have been linked to arterial stiffness. Second, increased arterial stiffness leads to an increase in cardiac ventricular load, thereby reducing cardiac systolic performance and increasing myocardial oxygen demand. ${ }^{30}$ This may lead to the progression of atherosclerosis, likely mediated by increased cycle stress leading to arterial wall thickening. ${ }^{31}$

Our results were consistent with previous studies which reported that baPWV is higher in patients with significant CAD compared to normal subjects. The baPWV measurements used for the diagnosis and assessment of CAD severity differ between studies. A previous study which reported that baPWV was limited in identifying CAD patients used the Gensini score to evaluate coronary atherosclerosis and showed that baPWV was not associated with calcification and severity of coronary arteries in high-risk patients. ${ }^{28}$ Another study reported that although baPWV was associated with significant CAD, it was neither associated significantly with the extent of CAD nor with the risk of revascularization ${ }^{32}$ and was limited in predicting the severity of CAD. In their study, the extent and severity of coronary atherosclerosis was evaluated by calculating the number of involved vessels. Xiong et al reported that baPWV was related to severity of CAD as assessed by angiography and the SYNTAX score. ${ }^{13}$ Although our results did not show a clinically relevant difference among the classified groups based on the number of involved vessels, we demonstrated that baPWV was well correlated with a significant increase in the severity and complexity of CAD as evaluated by the SYNTAX score. In addition, the present study was based on older patients with chest pain who visited the outpatient clinic, whose pain was documented as angina, and who underwent a stress test. Based on these considerations, we suggest that our results are clinically applicable in daily practice. The area under ROC curve was 0.639 in predicting coronary artery stenosis. The cutoff value of baPWV at $1,874 \mathrm{~cm} / \mathrm{s}$ had a sensitivity of $60.1 \%$, specificity of $70.8 \%$, positive predictive value of $87.0 \%$, and negative predictive value of $23.6 \%$.

\section{Limitations}

This study had some limitations. First, subjects undergoing CAG who visited a single center do not represent the entire population. Second, due to the cross-sectional design, it was impossible to assess the causality between baPWV and CAD severity, complexity, and clinical outcome. Third, the echocardiography and SYNTAX score were performed by different cardiologists. Because this is a retrospective analysis, the measurement could not be really double blinded. Fourth, many subjects were taking various medications, including antihypertensive drugs and lipid-lowering drugs, that may exert significant influences on baPWV. It is difficult to evaluate how exactly those medications influenced the numbers for baPWV. Finally, because the study population consisted of older patients with coronary heart disease, the cutoff value of baPWV does not represent all populations.

\section{Conclusion}

In this study, we showed that arterial stiffness determined by baPWV was associated independently with CAD severity, as assessed by angiography and the SYNTAX score in older patients with chest pain. Our data suggested that increased arterial stiffness assessed by baPWV is associated with the severity and presence of CAD in older patients.

\section{Disclosure}

The authors report no conflicts of interest in this work.

\section{References}

1. Goff DC Jr, Lloyd-Jones DM, Bennett G, et al; American College of Cardiology/American Heart Association Task Force on Practice Guidelines. 2013 ACC/AHA guideline on the assessment of cardiovascular risk: a report of the American College of Cardiology/American Heart Association Task Force on Practice Guidelines. Circulation. 2014; 129(25 suppl 2):S49-S73. 
2. Yamashina A, Tomiyama H, Takeda K, et al. Validity, reproducibility, and clinical significance of noninvasive brachial-ankle pulse wave velocity measurement. Hypertens Res. 2002;25(3):359-364.

3. Sipilä K, Koivistoinen T, Moilanen L, et al. Metabolic syndrome and arterial stiffness: the Health 2000 Survey. Metabolism. 2007;56(3): 320-326.

4. Kitahara T, Ono K, Tsuchida A, et al. Impact of brachial-ankle pulse wave velocity and ankle-brachial blood pressure index on mortality in hemodialysis patients. Am J Kidney Dis. 2005;46(4):688-696.

5. Morimoto S, Yurugi T, Aota Y, et al. Prognostic significance of anklebrachial index, brachial-ankle pulse wave velocity, flow-mediated dilation, and nitroglycerin-mediated dilation in end-stage renal disease. Am J Nephrol. 2009;30(1):55-63.

6. Tomiyama H, Koji Y, Yambe M, et al. Brachial - ankle pulse wave velocity is a simple and independent predictor of prognosis in patients with acute coronary syndrome. Circ J. 2005;69(7):815-822.

7. Nakamura M, Yamashita T, Yajima J, et al; Shinken Database Study Group. Brachial-ankle pulse wave velocity as a risk stratification index for the short-term prognosis of type 2 diabetic patients with coronary artery disease. Hypertens Res. 2010;33(10):1018-1024.

8. Meguro T, Nagatomo Y, Nagae A, et al. Elevated arterial stiffness evaluated by brachial-ankle pulse wave velocity is deleterious for the prognosis of patients with heart failure. Circ J. 2009;73(4):673-680.

9. Vlachopoulos C, Aznaouridis K, Terentes-Printzios D, Ioakeimidis N, Stefanadis C. Prediction of cardiovascular events and all-cause mortality with brachial-ankle elasticity index: a systematic review and metaanalysis. Hypertension. 2012;60(2):556-562.

10. Nam HJ, Jung IH, Kim J, et al. Association between brachial-ankle pulse wave velocity and occult coronary artery disease detected by multidetector computed tomography. Int J Cardiol. 2012;157(2):227-232.

11. Kullo IJ, Bielak LF, Turner ST, Sheedy PF 2nd, Peyser PA. Aortic pulse wave velocity is associated with the presence and quantity of coronary artery calcium: a community-based study. Hypertension. 2006;47(2): 174-179

12. Laurent S, Boutouyrie P, Asmar R, et al. Aortic stiffness is an independent predictor of all-cause and cardiovascular mortality in hypertensive patients. Hypertension. 2001;37(5):1236-1241.

13. Xiong Z, Zhu C, Zheng Z, et al. Relationship between arterial stiffness assessed by brachial-ankle pulse wave velocity and coronary artery disease severity assessed by the SYNTAX score. J Atheroscler Thromb. 2012;19(11):970-976.

14. Bramwell JC, Hill AV. Velocity of transmission of the pulse wave. Lancet. 1922;199:2.

15. Avolio AP, Chen SG, Wang RP, Zhang CL, Li MF, O'Rourke MF. Effects of aging on changing arterial compliance and left ventricular load in a northern Chinese urban community. Circulation. 1983;68(1):50-58.

16. Schiller NB, Shah PM, Crawford M, et al. Recommendations for quantitation of the left ventricle by two-dimensional echocardiography. American Society of Echocardiography Committee on Standards, Subcommittee on Quantitation of Two-Dimensional Echocardiograms. J Am Soc Echocardiogr. 1989;2(5):358-367.
17. Devereux RB, Reichek N. Echocardiographic determination of left ventricular mass in man. Anatomic validation of the method. Circulation. 1977;55(4):613-618.

18. Safar ME, Levy BI, Struijker-Boudier H. Current perspectives on arterial stiffness and pulse pressure in hypertension and cardiovascular diseases. Circulation. 2003;107(22):2864-2869.

19. Safar ME, Henry O, Meaume S. Aortic pulse wave velocity: an independent marker of cardiovascular risk. Am J Geriatr Cardiol. 2002;11(5): 295-298.

20. Washida N, Wakino S, Hayashi K, Kuwahara T, Itoh H. Brachial-ankle pulse wave velocity predicts silent cerebrovascular diseases in patients with end-stage renal diseases. J Atheroscler Thromb. 2010;17(2): $165-172$.

21. Yamashina A, Tomiyama H, Arai T, et al. Brachial-ankle pulse wave velocity as a marker of atherosclerotic vascular damage and cardiovascular risk. Hypertens Res. 2003;26(8):615-622.

22. Tsuchikura S, Shoji T, Kimoto E, et al. Brachial-ankle pulse wave velocity as an index of central arterial stiffness. J Atheroscler Thromb. 2010;17(6) 658-665.

23. Serruys PW, Onuma Y, Garg S, et al. Assessment of the SYNTAX score in the Syntax study. Euro Intervention. 2009;5(1):50-56.

24. Valgimigli M, Serruys PW, Tsuchida K, et al; ARTS II. Cyphering the complexity of coronary artery disease using the SYNTAX score to predict clinical outcome in patients with three-vessel lumen obstruction undergoing percutaneous coronary intervention. Am J Cardiol. 2007; 99(8):1072-1081.

25. Duprez DA, Cohn JN. Monitoring vascular health beyond blood pressure. Curr Hypertens Rep. 2006;8(4):287-291.

26. Nigam A, Mitchell GF, Lambert J, Tardif JC. Relation between conduit vessel stiffness (assessed by tonometry) and endothelial function (assessed by flow-mediated dilatation) in patients with and without coronary heart disease. Am J Cardiol. 2003;92(4):395-399.

27. Widlansky ME, Gokce N, Keaney JF Jr, Vita JA. The clinical implications of endothelial dysfunction. J Am Coll Cardiol. 2003;42(7):1149-1160.

28. Kim HJ, Nam JS, Park JS, et al. Usefulness of brachial-ankle pulse wave velocity as a predictive marker of multiple coronary artery occlusive disease in Korean type 2 diabetes patients. Diabetes Res Clin Pract. 2009; 85(1):30-34

29. Xu Y, Wu Y, Li J, et al. The predictive value of brachial-ankle pulse wave velocity in coronary atherosclerosis and peripheral artery diseases in urban Chinese patients. Hypertens Res. 2008;31(6):1079-1085.

30. Dart AM, Kingwell BA. Pulse pressure - a review of mechanisms and clinical relevance. J Am Coll Cardiol. 2001;37(4):975-984.

31. Shyy JY, Chien S. Role of integrins in endothelial mechanosensing of shear stress. Circ Res. 2002;91(9):769-775.

32. Chae MJ, Jung IH, Jang DH, et al. The brachial ankle pulse wave velocity is associated with the presence of significant coronary artery disease but not the extent. Korean Circ J. 2013;43(4):239-245.
Clinical Interventions in Aging

\section{Publish your work in this journal}

Clinical Interventions in Aging is an international, peer-reviewed journal focusing on evidence-based reports on the value or lack thereof of treatments intended to prevent or delay the onset of maladaptive correlates of aging in human beings. This journal is indexed on PubMed Central, MedLine,
CAS, Scopus and the Elsevier Bibliographic databases. The manuscript management system is completely online and includes a very quick and fair peer-review system, which is all easy to use. Visit http://www.dovepress. com/testimonials.php to read real quotes from published authors. 